\title{
Coherent Magneto-Optical Effects in Topological Insulators: Excitation Near the Absorption Edge
}

\author{
Wang-Kong Tse \\ Department of Physics and Astronomy, Center for Materials for Information Technology, \\ The University of Alabama, Alabama 35487, USA
}

\begin{abstract}
We study coherent optics in topological insulator surface states with broken time-reversal symmetry and develop a theory for the dynamical Hall effect driven by intense electromagnetic field. The influence of optical Stark effect enters as nonlinear dependence on the optical field in the resulting Faraday $\theta_{F}$ and Kerr $\theta_{K}$ rotations. This nonlinear correction is found to decrease $\theta_{F}$ with the strength of the A.C. electric field, whereas $\theta_{K}$ exhibits a non-monotonic behavior. We also assess the effects of relaxation and dephasing on the Hall and magneto-optical responses when the frequency detuning is comparable to the inverse lifetime of the conduction electrons.

PACS numbers: 78.20.Ls,73.43.-f,75.85.+t,78.67.-n
\end{abstract}

\section{INTRODUCTION}

Topological insulators are materials with strong spinorbit coupling that host topologically protected and gapless surface states [1]. The surface states' electrons are massless Dirac fermions with a Dirac cone energy dispersion close to the band crossing point that corresponds to spin degeneracy. One intriguing property that follows from the removal of this spin degeneracy by time-reversal symmetry breaking is that a band gap opens up and the system exhibits a Hall response [2]. If the Fermi level falls within the band gap, the Hall effect is quantized with a Hall conductivity equal to half of the unit conductance $\sigma_{x y}=e^{2} / 2 h$. The half quantization is a consequence of the helical spin texture of the surface Dirac electrons wrapping half of the unit sphere when the lower surface band is fully filled. It may also be understood as a bulk magnetoelectric effect [3 [5] developed from an additional term $\propto \theta \boldsymbol{E} \cdot \boldsymbol{B}$ in the electromagnetic Lagrangian (where $\theta$ is called the axion coupling constant [6]). As a result of this quantized Hall effect, topological insulator surface states behave as a quantum anomalous Hall insulator (also called a Chern insulator) and give rise to strong magneto-optical responses. The Faraday $\theta_{F}$ and Kerr $\theta_{K}$ angles are predicted to display quantized values with $\theta_{F}$ quantized to multiples to the fine structure constant $\alpha=1 / 137$ and $\theta_{K}$ quantized to a full-quarter rotation $\pi / 2$ in topological insulator thin films [7/ 9 . Soon after theoretical predictions, colossal values of up to $60^{\circ}$ of the Kerr effect [10] in $\mathrm{Bi}_{2} \mathrm{Se}_{3}$ topological insulator thin films were measured. Recently, three groups have independently reported measurements of the predicted quantization of the Faraday effect [11 14. The anomalous Hall transport is, naturally, a linear response effect and therefore the resulting topological Faraday and Kerr angles are independent of the optical field strength, corresponding to a regime where the electromagnetic field can be treated as a perturbation.

Strong A.C. fields lead to interesting nonlinear optical properties and are studied extensively in conventional semiconductors [15]. For example, strong electromag- netic radiation acts to renormalize the conduction and valence bands in the saturation state of a semiconductor with the optically dressed electrons and holes behaving as new quasiparticles [16. Optical nonlinearity and optically-induced coherent effects pose a new frontier in recently discovered materials including topological insulators, where coherent control of topological properties might be possible. In particular, the influence of strong radiation on the magneto-transport properties in topological insulators presents an interesting and unexplored area of investigation that could lead to new insights on the interplay between light and band topology.

The present paper attempts to address some of the questions along this direction by generalizing our previous considerations on the magneto-transport and magneto-optical properties of topological insulators to strong electromagnetic fields. We shall focus on the small detuning regime where the light frequency is close to the absorption threshold, so that the rotating wave approximation (RWA) remains a viable strategy of solution while allowing the effects of strong electromagnetic fields to be studied non-perturbatively.

An outline of the paper is as follows. We first lay out our model for topological insulator surface states coupled to electromagnetic fields in Sec. III In Sec. III. we consider the coherent regime in the absence of damping and discuss the effects of strong fields on the resulting non-equilibrium quasiparticle distribution functions and energy dispersions. We then proceed to calculate the dynamical longitudinal and Hall current responses in Sec. IV] In Sec. V] we formulate the equations that incorporates nonlinear optical effects in the transmission coefficients and calculate the nonlinear magneto-optical Faraday and Kerr effects. Finally, we consider the effects of relaxation and dephasing on the dynamical current responses and magneto-optical effects in the regime where the detuning is comparable to damping rates in Sec. VI. 


\section{MODEL}

Under time-reversal symmetry breaking, topological insulator surface states are described by the $2 \times 2$ massive Dirac Hamiltonian

$$
H^{0}=\frac{1}{2}\left(\varepsilon_{k+}-\varepsilon_{k-}\right)\left[\begin{array}{cc}
\cos \theta_{k} & e^{-i \phi_{k}} \sin \theta_{k} \\
e^{i \phi_{k}} \sin \theta_{k} & -\cos \theta_{k}
\end{array}\right],
$$

where $\varepsilon_{k \pm}= \pm \alpha_{k}$ are the conduction $(+)$ and valence band (-) energies with $\alpha_{k}=\sqrt{(v k)^{2}+\Delta^{2}}, \tan \phi_{k}=$ $k_{y} / k_{x}$ is the azithmuthal angle of the electron momentum, $\theta_{k}$ is the polar angle with $\cos \theta_{k}=\Delta / \alpha_{k}$ and $\sin \theta_{k}=v k / \alpha_{k}$. Throughout this work, we set $\hbar=1$ unless otherwise specified. The discrete binary degrees of freedom describe electron spins. $\Delta$ is the Zeeman field acting on the electron spins, which gives the Dirac surface states a band gap of $2 \Delta$. This Zeeman field can be induced by exchange coupling to the topological insulator surface states or by doping with magnetic atoms.

We introduce a set of spin unit vectors $\left\{\hat{\boldsymbol{\alpha}}_{k}, \hat{\boldsymbol{\beta}}_{k}, \hat{\gamma}_{k}\right\}$ (the reader is referred to the Appendix for their definitions), and define the Pauli matrices in the new basis $\left(\sigma_{\alpha}, \sigma_{\beta}, \sigma_{\gamma}\right)=\boldsymbol{\sigma} \cdot\left(\hat{\boldsymbol{\alpha}}_{k}, \hat{\boldsymbol{\beta}}_{k}, \hat{\boldsymbol{\gamma}}_{k}\right)$ that satisfy the commutation relation $\left[\sigma_{\alpha}, \sigma_{\beta}\right]=2 i \sigma_{\gamma}$. $\hat{\boldsymbol{\alpha}}_{k}$ describes the local orientation of the electron spin at momentum $\boldsymbol{k}$. The Hamiltonian can then be expressed in the form of a Zeeman coupling term $H^{0}=\mathcal{B}_{k}^{0} \cdot \boldsymbol{\sigma} / 2$ with $\mathcal{B}_{k}^{0}=2 \alpha_{k} \hat{\boldsymbol{\alpha}}_{k}$ taking on the meaning of an effective magnetic field.

Initially, our system is in an unexcited state with a fully filled valance band and an empty conduction band. A linearly polarized light is then illuminated onto the topological insulator with an electric field $\boldsymbol{E}=E_{0} \cos \omega t \hat{\boldsymbol{e}}_{x}$ and a polarization state indicated by the unit vector along $x$ direction $\hat{\boldsymbol{e}}_{x}$. The electron-photon interaction Hamiltonian $H^{p}(t)=\left(e E_{0} v / \omega\right) \sin \omega t \sigma_{x}$ can be similarly expressed as a Zeeman coupling term $H^{p}(t)=\mathcal{B}_{k}^{p}(t) \cdot \boldsymbol{\sigma} / 2$ with a lightinduced effective magnetic field

$$
\begin{aligned}
\mathcal{B}_{k}^{p}= & \Lambda \sin \omega t\left(\sin \theta_{k} \cos \phi_{k} \hat{\boldsymbol{\alpha}}_{k}-\sin \phi_{k} \hat{\boldsymbol{\beta}}_{k}\right. \\
& \left.-\cos \theta_{k} \cos \phi_{k} \hat{\gamma}_{k}\right),
\end{aligned}
$$

where $\Lambda=2 e E_{0} v / \omega$ is the strength of the interband transition matrix element and corresponds to the energy acquired by an electron driven by the $A$. C. field over a half period.

We consider optical response of the system under irradiation with intense off-resonant light having a frequency $\omega<2 \Delta$ and a small detuning $\delta \equiv 2 \Delta-\omega \ll 2 \Delta$. The regime of strong electromagnetic fields is characterized by $\Lambda \gg 1 / \tau$, where $\tau$ is the electron lifetime. The dynamics of the system's $2 \times 2$ density matrix $\rho_{k}(t)$ is governed by the quantum kinetic equation

$$
\frac{\partial \rho_{k}}{\partial t}+i\left[H, \rho_{k}\right]=I_{\mathrm{c}}\left(\rho_{k}, t\right)
$$

where $H(t)=H^{0}+H^{p}(t)$ is the total Hamiltonian of the system, and the collision integral $I_{\mathrm{c}}\left(\rho_{k}, t\right)$ takes into account damping effects from relaxation and dephasing. In the following, (1) we first study and elucidate the main physics in the coherent regime where the detuning $\delta \gg$ $1 / \tau$ in Secs. III V (2) we then consider the case $\delta \lesssim$ $1 / \tau$ where the effects of relaxation and dephasing become non-negligible in Sec. VI

\section{COHERENT LIGHT-DRIVEN SPIN DYNAMICS}

In the coherent regime when detuning $\delta \gg 1 / \tau$, one can ignore the collision integral in the kinetic equation Eq. (3). To analyze the spin dynamics, we resolve the density matrix into its charge $n_{k}$ and $\operatorname{spin} \boldsymbol{S}_{k}=$ $S_{k}^{\alpha} \hat{\boldsymbol{\alpha}}_{k}+S_{k}^{\beta} \hat{\boldsymbol{\beta}}_{k}+S_{k}^{\gamma} \hat{\gamma}_{k}$ sectors so that $\rho_{k}=n_{k} \mathbb{I}+\boldsymbol{S}_{k} \cdot \boldsymbol{\sigma} / 2$, where $S_{k}^{\alpha}, S_{k}^{\beta}, S_{k}^{\gamma}$ are real. This gives the Bloch equation governing the dynamics of the spin density matrix due to the total effective magnetic field $\mathcal{B}_{k}=\mathcal{B}_{k}^{0}+\mathcal{B}_{k}^{p}$,

$$
\frac{\partial \boldsymbol{S}_{k}}{\partial t}+\boldsymbol{S}_{k} \times \mathcal{B}_{k}=0
$$

with the initial condition $\boldsymbol{S}_{k}(t=0)=-\hat{\boldsymbol{\alpha}}_{k}$. Eq. (4) implies that the magnitude of the $\boldsymbol{S}_{k}$ is a constant of motion with $\left|\boldsymbol{S}_{k}\right|=1$. The component of the density matrix $S_{k}^{\alpha}$ along $\hat{\boldsymbol{\alpha}}_{k}$ corresponds to population difference due to interband transitions whereas the components $S_{k}^{\beta}, S_{k}^{\gamma}$ along the orthogonal directions $\hat{\boldsymbol{\beta}}_{k}, \hat{\gamma}_{k}$ corresponds to interband coherence.

It is convenient to define the longitudinal $\hat{\boldsymbol{\alpha}}_{k}^{\mathrm{L}}=\hat{\boldsymbol{\alpha}}_{k}$ and transverse $\hat{\boldsymbol{\alpha}}_{k}^{\mathrm{T}}=\hat{\boldsymbol{\beta}}_{k}-i \hat{\boldsymbol{\gamma}}_{k}$ spin vectors and transform our reference frame into the rotating frame at the laser frequency. In the rotating frame then, the longitudinal $S_{k}^{\mathrm{L}}$ and transverse spin density matrices $S_{k}^{\mathrm{T}}$ are given as

$$
\begin{aligned}
S_{k}^{\mathrm{L}} & \equiv \boldsymbol{S}_{k} \cdot \hat{\boldsymbol{\alpha}}_{k}^{\mathrm{L}}, \\
S_{k}^{\mathrm{T}} & \equiv e^{i \omega t} \boldsymbol{S}_{k} \cdot \hat{\boldsymbol{\alpha}}_{k}^{\mathrm{T}},
\end{aligned}
$$

whereas the longitudinal $\mathcal{B}_{k}^{\mathrm{L}}$ and transverse $\mathcal{B}_{k}^{\mathrm{T}}$ components of the total effective magnetic field are defined as

$$
\begin{aligned}
\mathcal{B}_{k}^{\mathrm{L}} & =\mathcal{B}_{k} \cdot \hat{\boldsymbol{\alpha}}_{k}^{\mathrm{L}}-\omega, \\
\mathcal{B}_{k}^{\mathrm{T}} & =e^{i \omega t} \boldsymbol{\mathcal { B }}_{k} \cdot \hat{\boldsymbol{\alpha}}_{k}^{\mathrm{T}} .
\end{aligned}
$$

It then follows from the above Eqs. (7)-(8) that

$$
\begin{aligned}
& \mathcal{B}_{k}^{\mathrm{L}}=2 \alpha_{k}-\omega+\hat{\boldsymbol{\alpha}}_{k}^{\mathrm{L}} \cdot \mathcal{B}_{k}^{p}, \\
& \mathcal{B}_{k}^{\mathrm{T}}=e^{i \omega t} \hat{\boldsymbol{\alpha}}_{k}^{\mathrm{T}} \cdot \boldsymbol{\mathcal { B }}_{k}^{p} .
\end{aligned}
$$

In terms of these new variables, we can recast Eq. (4) as

$$
\begin{aligned}
i \frac{\partial S_{k}^{\mathrm{L}}}{\partial t} & =\frac{1}{2}\left[\left(S_{k}^{\mathrm{T}}\right)^{*} \mathcal{B}_{k}^{\mathrm{T}}-S_{k}^{\mathrm{T}}\left(\mathcal{B}_{k}^{\mathrm{T}}\right)^{*}\right], \\
i \frac{\partial S_{k}^{\mathrm{T}}}{\partial t} & =S_{k}^{\mathrm{T}} \mathcal{B}_{k}^{\mathrm{L}}-S_{k}^{\mathrm{L}} \mathcal{B}_{k}^{\mathrm{T}},
\end{aligned}
$$

supplemented by the initial conditions $S_{k}^{\mathrm{L}}(t=0)=-1$ and $S_{k}^{\mathrm{T}}(t=0)=0$ corresponding to a unexcited system 
with a fully filled valance band. The above equations are equivalent to the semiconductor Bloch equations [15] in the conduction-valence band representation with Hamiltonian $H_{k, \mu \nu}$ and density matrix $\rho_{k, \mu \nu}(\mu, \nu= \pm$ denoting the conduction and valence bands): the longitudinal and transverse components of the spin density matrix are respectively related to the population difference $S_{k}^{\mathrm{L}}=\rho_{k,++}-\rho_{k,--}$ and interband coherence $S_{k}^{\mathrm{T}}=-2 i \rho_{k,+-} e^{i \omega t}$ between the two bands; whereas the longitudinal and transverse components of the effective magnetic field are respectively related to the band energy difference (including the intraband dipole matrix element) $\mathcal{B}_{k}^{\mathrm{L}}=H_{k,++}-H_{k,--}$ and interband dipole matrix element $-2 i H_{k,+-} e^{i \omega t}=\mathcal{B}_{k}^{\mathrm{T}}$.

We are interested in the optical response at the same frequency as the incident field. Since the detuning $\delta \ll 2 \Delta$ is small, we work in the rotating wave approximation (RWA) keeping only the resonant terms. The longitudinal and transverse components of the effective magnetic field then becomes $\mathcal{B}_{k}^{\mathrm{L}}=2 \alpha_{k}-\omega$ and $\mathcal{B}_{k}^{\mathrm{T}}=-\Lambda\left(\cos \theta_{k} \cos \phi_{k}+i \sin \phi_{k}\right) / 2$. The quantity $\left|\mathcal{B}_{k}^{\mathrm{T}}\right|^{2}$, corresponding to the squared amplitude of the interband transition matrix element, appears frequently in our following discussions; for convenience, we shall denote the momentum-dependent factor in $\left|\mathcal{B}_{k}^{\mathrm{T}}\right|^{2}$ as $X_{k}^{2}=\cos ^{2} \theta_{k} \cos ^{2} \phi_{k}+\sin ^{2} \phi_{k}$. When the switch-on time of the laser pulse is much longer than $\Lambda^{-1}$, the switching process is adiabatic and the solution to Eqs. (11)-(12) corresponds to the spin dynamics adiabatically following the effective magnetic field. Eqs. (11)- $(12)$ then implies that $\left(S_{k}^{\mathrm{T}} / \mathcal{B}_{k}^{\mathrm{T}}\right)^{*}=\left(S_{k}^{\mathrm{T}} / \mathcal{B}_{k}^{\mathrm{T}}\right)=\left(S_{k}^{\mathrm{L}} / \mathcal{B}_{k}^{\mathrm{L}}\right) \equiv \mathcal{C}$, where $\mathcal{C}$ is a real constant. By virtue of the normalization condition $\left|S_{k}^{\mathrm{L}}\right|^{2}+\left|S_{k}^{\mathrm{T}}\right|^{2}=1$, the spin density matrix components are found to be

$$
\begin{aligned}
S_{k}^{\mathrm{L}} & =-\frac{\left|\mathcal{B}_{k}^{\mathrm{L}}\right|}{\sqrt{\left|\mathcal{B}_{k}^{\mathrm{L}}\right|^{2}+\left|\mathcal{B}_{k}^{\mathrm{T}}\right|^{2}}}, \\
S_{k}^{\mathrm{T}} & =-\frac{\mathcal{B}_{k}^{\mathrm{T}}}{\sqrt{\left|\mathcal{B}_{k}^{\mathrm{L}}\right|^{2}+\left|\mathcal{B}_{k}^{\mathrm{T}}\right|^{2}}} .
\end{aligned}
$$

The above distribution functions Eqs. (13)-(14) highlight the semiconductor optical Stark effect 17. under the adiabatic switch-on condition. Illuminated with a strong optical field, the conduction and valence band states become mixed by the dipole matrix element with the system becoming a coherent ground state of photon dressed electron-hole pairs. These dressed electron-hole pairs constitute the new quasiparticles of the irradiated system with energy dispersions $\pm E_{k}= \pm \sqrt{\left|\mathcal{B}_{k}^{\mathrm{L}}\right|^{2}+\left|\mathcal{B}_{k}^{\mathrm{T}}\right|^{2}}$ in the rotating frame. The leading-order energy shift is $\sim E_{0}^{2}$ characteristic of the optical Stark effect. From Eq. (13), the conduction band distribution function is found to be $\rho_{k+}=(1 / 2)\left(1+S_{k}^{\mathrm{L}}\right)$. For the case of linearly polarized light illumination on a 2D Dirac electron system, $\mathcal{B}_{k}^{\mathrm{T}}$ depends explicitly on $\phi_{k}$, and the renormalized band energies as well as the conduction and valance

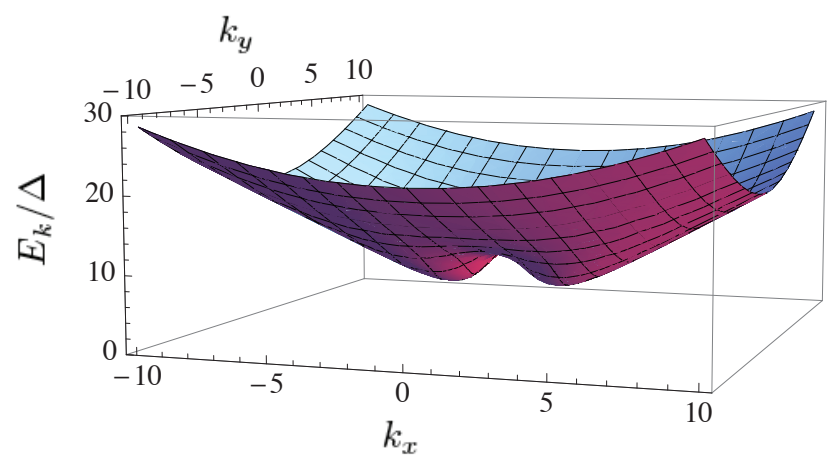

FIG. 1: (Color online). 3D plot of the renormalized conduction band energy $E_{k}$ (scaled by the gap $\Delta$ ) in the rotating frame. The surface Dirac gap $\Delta=80 \mathrm{meV}, \omega=60 \mathrm{meV}$, $E=150 \mathrm{MVm}^{-1}$.

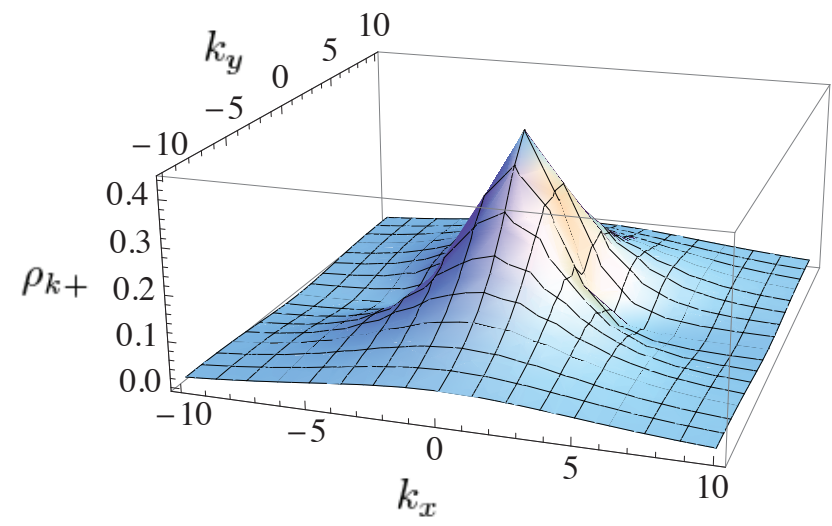

FIG. 2: (Color online). 3D plot of the conduction band distribution function $f_{k+}$ in the rotating frame. Values for $\Delta$ and $\omega$ are the same as in Fig. 1

band distribution functions are anisotropic in the momentum space as depicted in Figs. 1. 2, Although light is off-resonance, in the strong-field regime there is always a finite electron population in the conduction band due to nonlinear effects.

\section{DYNAMICS OF CURRENT RESPONSE}

The current density in the system due to the $A$.C. field is given by $\boldsymbol{J}(t)=\sum_{k} \operatorname{tr}\left\{\boldsymbol{j}_{k}(t) f_{k}(t)\right\}$, where $\boldsymbol{j}_{k}(t)=$ $\partial H(t) / \partial \boldsymbol{k}$ is the single-particle current operator. The current density can be written in terms of the effective magnetic field and the spin density matrix as

$$
\boldsymbol{J}(t)=\left.\frac{1}{2} \sum_{k} \frac{\partial \boldsymbol{B}_{k^{\prime}} \cdot \boldsymbol{S}_{k}}{\partial \boldsymbol{k}^{\prime}}\right|_{\boldsymbol{k}^{\prime}=\boldsymbol{k}} .
$$

Eqs. 13 - 14 are obtained in the rotating frame. In order to compute the current from Eq. (15), we transform back into the stationary frame. Then, the resonant contribution of the effective magnetic field 
$\left.\boldsymbol{\mathcal { R }}_{k} \equiv \mathcal{B}_{k}^{p}\right|_{\text {resonance }}$ due to electron-photon coupling can be written as $\boldsymbol{\mathcal { R }}_{k}=\mathcal{R}_{k}^{\beta} \hat{\boldsymbol{\beta}}_{k}+\mathcal{R}_{k}^{\gamma} \hat{\gamma}_{k}$, where $\mathcal{R}_{k}^{\beta}=$ $\operatorname{Re}\left\{\mathcal{B}_{k}^{\mathrm{T}} e^{-i \omega t}\right\}=(\Lambda / 2)\left(\cos \theta_{k} \cos \phi_{k} \cos \omega t+\sin \phi_{k} \sin \omega t\right)$ and $\mathcal{R}_{k}^{\gamma}=-\operatorname{Im}\left\{\mathcal{B}_{k}^{\mathrm{T}} e^{-i \omega t}\right\}=(\Lambda / 2)\left(\cos \theta_{k} \cos \phi_{k} \sin \omega t-\right.$ $\left.\sin \phi_{k} \cos \omega t\right)$. The spin density matrix from Eqs. (13)(14) is now expressed in the stationary frame as

$$
\boldsymbol{S}_{k}=-f_{k}(\Lambda)\left[\left(2 \alpha_{k}-\omega\right) \hat{\boldsymbol{\alpha}}_{k}+\mathcal{R}_{k}^{\beta} \hat{\boldsymbol{\beta}}_{k}+\mathcal{R}_{k}^{\gamma} \hat{\gamma}_{k}\right],
$$

where

$$
f_{k}(\Lambda)=\frac{1}{\sqrt{\left.\left(2 \alpha_{k}-\omega\right)^{2}+\left(\Lambda X_{k} / 2\right)^{2}\right)}},
$$

where $X_{k}^{2}=\cos ^{2} \theta_{k} \cos ^{2} \phi_{k}+\sin ^{2} \phi_{k}$ as defined in the paragraph before Eqs. (13)-(14). As usual, the sinuisoidal time dependence can be taken care of by defining complex quantities associated with the exponential time factor $e^{-i \omega t}$. We define the complex current density $\mathcal{J}$ through $\boldsymbol{J}(t)=\operatorname{Re}\left\{\mathcal{J} e^{-i \omega t}\right\}$. Using Eq. 15, we find the longitudinal and Hall components of the current density in response to the $A$. $C$. field

$$
\begin{aligned}
& \mathcal{J}_{x}=-i \frac{e^{2} E_{0} v^{2}}{2} \sum_{k} \frac{f_{k}(\Lambda)}{\alpha_{k}} X_{k}^{2}, \\
& \mathcal{J}_{y}=\frac{e^{2} E_{0} v^{2} \Delta}{2} \sum_{k} \frac{f_{k}(\Lambda)}{\alpha_{k}^{2}} .
\end{aligned}
$$

\section{A. Weak Fields: Relation to Linear Response Regime}

To see how the strong field regime is connected to the linear response regime, it is instructive to expand the results Eqs. (18)- $(19)$ to leading order in powers of $E_{0}$. For weak fields such that $\Lambda \ll \delta$, we expand Eqs. (18)$(19)$ in $\Lambda / \delta$ up to the leading order. The integrals can be calculated analytically and yield

$$
\begin{aligned}
\mathcal{J}_{x}= & -i \frac{e^{2} E_{0}}{8 \pi}\left[\frac{\Delta^{2}}{\omega}\left(\frac{1}{\varepsilon_{c}}-\frac{1}{\Delta}\right)\right. \\
& \left.-2\left(\frac{\Delta}{\omega}\right)^{2} \ln \left|\frac{\varepsilon_{c}}{\omega}\right|+\frac{1}{2}\left(1+4 \frac{\Delta^{2}}{\omega^{2}}\right) \ln \left|\frac{\omega-2 \varepsilon_{c}}{\omega-2 \Delta}\right|\right], \\
\mathcal{J}_{y}= & \frac{e^{2} E_{0} \Delta}{4 \pi \omega}\left(\ln \left|\frac{\omega-2 \varepsilon_{c}}{\varepsilon_{c}}\right|-\ln \left|\frac{\omega-2 \Delta}{\Delta}\right|\right),
\end{aligned}
$$

where $\varepsilon_{c}$ is an ultraviolet energy cut-off that corresponds the bandwidth of the surface Dirac bands, taken to be the bulk energy gap of the topological insulator. We emphasize that the expressions for $\mathcal{J}_{x}$ and $\mathcal{J}_{y}$ above are only valid for non-zero frequencies $\omega \approx 2 \Delta$. One cannot arrive at the $D$.C. limit by taking $\omega \rightarrow 0$ in the above expressions because the counter-rotating contributions, which are ignored in RWA, become comparable at $\omega \rightarrow 0$ to the rotating (i.e., resonant) contributions retained in the
RWA. To recover the D.C. limit, and indeed the full linear response optical conductivity, one needs to add in the counter-rotating contributons. To illustrate this point, we can explicitly take the $\omega \rightarrow 0$ limit and see what happens. First, $J_{x}(t)$ vanishes in this limit as expected because $e^{-i \omega t} \rightarrow 1$ and Eq. 200 is purely imaginary. Then, expanding up to leading order in $\omega / \Delta$ in Eq. 211, we find that $\mathcal{J}_{y}=e^{2} E_{0} / 8 \pi$ corresponding to a Hall conductivity of $\sigma_{x y}=e^{2} / 4 h$. Interestingly, in the rotating-wave approximation where the counter-rotating contributions to the effective magnetic field are discarded, the zerofrequency Hall response from Eq. 21) amounts to $1 / 2$ of the well-known quantized Hall conductivity $\sigma_{x y}=e^{2} / 2 h$ of the Dirac model in the linear response regime. Indeed, it can be easily checked that our weak-field results Eqs. (20)-(21) correspond exactly to the resonant contribution of the established expressions of dynamical conductivities of the Dirac model [7. Adding in also the counter-rotating contribution, which is separately due to an effective magnetic field $\left.\boldsymbol{B}_{k}^{p}\right|_{\text {anti-resonance }}=\mathcal{R}_{k}(-\omega)$ (noting that $\Lambda \rightarrow-\Lambda$ also under $\omega \rightarrow-\omega$ in $\boldsymbol{\mathcal { R }}_{k}$ since $\Lambda$ is dependent on $\omega$ ), yields the full optical conductivities of the Dirac model.

\section{B. Strong Fields}

For strong fields with $\Lambda \gg \delta$, we have

$$
f_{k}(\Lambda) \simeq \frac{2}{\Lambda} \frac{1}{X_{k}}
$$

Eqs. 18)-19 can then be evaluated analytically yielding

$$
\begin{aligned}
\mathcal{J}_{x}= & -i \frac{e \omega}{2 \pi^{2} v \varepsilon_{c}} \\
& \times\left[\varepsilon_{c}^{2} \mathbb{E}\left(\sqrt{1-\frac{\Delta^{2}}{\varepsilon_{c}^{2}}}\right)-\Delta^{2} \mathbb{K}\left(\sqrt{1-\frac{\Delta^{2}}{\varepsilon_{c}^{2}}}\right)\right], \\
\mathcal{J}_{y}= & \frac{e \omega \Delta}{2 \pi^{2} v}\left[\mathbb{K}\left(\sqrt{1-\frac{\Delta^{2}}{\varepsilon_{c}^{2}}}\right)-\mathbb{E}\left(\sqrt{1-\frac{\Delta^{2}}{\varepsilon_{c}^{2}}}\right)\right],
\end{aligned}
$$

where $\mathbb{K}, \mathbb{E}$ are the complete elliptic integrals of the first and second kind, respectively. At high fields, therefore, the longitudinal and Hall currents both saturate to values independent of the incident field.

We evaluate Eqs. (18)-(19) numerically and display the computed current densities in Fig. 3 . The longitudinal and Hall currents increase linearly with the electric field and saturate at high $E_{0}$ values, as predicted from the analytic results Eqs. (20)-(21) and Eqs. (23)-(24). We also see that increasing detuning has the effect of decreasing the current amplitudes, consistent with the behavior that the saturation currents are proportional to $\omega$ in Eqs. (23)(24). 


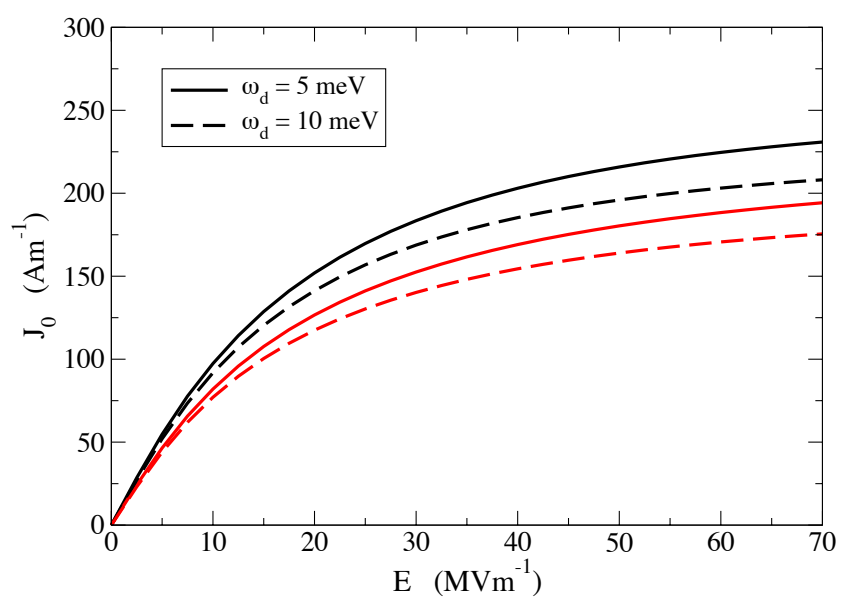

FIG. 3: (Color online) Amplitudes $J_{x 0}$ (dark/black) and $J_{y 0}$ (grey/red) of the longitudinal and Hall current densities $J_{x 0}=$ $\left|\mathcal{J}_{x}\right|$ and $J_{y 0}=\left|\mathcal{J}_{y}\right|$ as a function of the electric field $E_{0}$ [Eqs. [18]-[19] ] for different values of detuning $\omega_{\mathrm{d}}=5 \mathrm{meV}$ and $10 \mathrm{meV}$. The surface Dirac gap $\Delta=50 \mathrm{meV}$ and Dirac band cutoff energy $\varepsilon_{\mathrm{c}}=175 \mathrm{meV}$.

\section{NONLINEAR MAGNETO-OPTICAL FARADAY AND KERR EFFECTS}

The usual Fresnel relations, which relate the transmitted and reflected electric fields to the incident field, are derived assuming linear response to the incident light field. Also, the scattering (or, equivalently, transfer) matrix formalism assumes linearity between the scattered fields and the incident field. In order to take into account non-perturbative electric field effects in the current response, these two standard approaches are not applicable and we need to formulate the problem of nonlinear magneto-optical response differently as follows.

Consider light illuminated along the $z$ direction on the topological insulator surface that is located at $z=0$. The two regions $z<0$ and $z>0$ are labeled by $j=\mathrm{I}$, II with dielectric constant $\epsilon_{j}$. Expressing, as usual, the real electric and magnetic fields as complex vectors $\boldsymbol{E}=$ $\operatorname{Re}\left\{\mathcal{E} e^{-i \omega t}\right\}$ and $\boldsymbol{H}=\operatorname{Re}\left\{\mathcal{H} e^{-i \omega t}\right\}$ respectively, we write the electric field in the form

$$
\tilde{\mathcal{E}}^{j}=e^{i k_{j} z}\left[\begin{array}{c}
\mathcal{E}_{x}^{t j} \\
\mathcal{E}_{y}^{t j}
\end{array}\right]+e^{-i k_{j} z}\left[\begin{array}{c}
\mathcal{E}_{x}^{r j} \\
\mathcal{E}_{y}^{r j}
\end{array}\right]
$$

where the tilde accents denote column vectors $\tilde{\mathcal{E}}=$ $\left[\begin{array}{ll}\mathcal{E}_{x} & \mathcal{E}_{y}\end{array}\right]^{\mathrm{T}}$, the superscripts ' $\mathrm{r}$ ' and 't' on $\mathcal{E}_{x, y}$ denote the reflected and transmitted field components, and $k_{j}=$ $\sqrt{\epsilon_{j}} k_{0}$ is the wavevector in region $j$ with dielectric constant $\epsilon_{j}, k_{0}=\omega / c$ and $c$ is the speed of light. The corresponding magnetic field is given by Faraday's law as

$$
\tilde{\mathcal{H}}^{j}=\sqrt{\epsilon_{j}}\left\{e^{i k_{j} z}\left[\begin{array}{c}
-\mathcal{E}_{y}^{t j} \\
\mathcal{E}_{x}^{t j}
\end{array}\right]+e^{-i k_{j} z}\left[\begin{array}{c}
\mathcal{E}_{y}^{r j} \\
-\mathcal{E}_{x}^{r j}
\end{array}\right]\right\},
$$

The electric and magnetic fields at the interface $z=0$ satisfy the electrodynamic boundary conditions $\tilde{\mathcal{E}}^{\mathrm{I}}=\tilde{\mathcal{E}}^{\mathrm{II}}$ and $-i \tau_{y}\left(\tilde{\mathcal{H}}^{\mathrm{II}}-\tilde{\mathcal{H}}^{\mathrm{I}}\right)=(4 \pi / c) \tilde{\mathcal{J}}$, where $\left(\tau_{x}, \tau_{y}, \tau_{z}\right)$ are Pauli matrices and $\tilde{\mathcal{J}}=\left[\begin{array}{ll}\mathcal{J}_{x} & \mathcal{J}_{y}\end{array}\right]^{\mathrm{T}}$ is the current density of the topological surface states at $z=0$.

We calculate the transmission and reflection coefficients due to incident light that is linearly polarized along $x, \tilde{\mathcal{E}}^{\mathrm{I}}=e^{i k_{0} z}\left[E_{0} 0\right]^{\mathrm{T}}$. For a single interface, the scattered field components in region I and region II correspond to the reflected and transmitted fields respectively, and to simplify the notation we shall drop the superscripts I and II with no danger of confusion. The transmission and reflection coefficents along the directions $\alpha=x, y$ are defined as $T_{\alpha}=\mathcal{E}_{\alpha}^{t} / E_{0}$ and $R_{\alpha}=\mathcal{E}_{\alpha}^{r} / E_{0}$, which are functions of $E_{0}$ in the nonlinear regime. From the electromagnetic boundary conditions we then obtain the following set of coupled nonlinear equations for $T_{x}$ and $T_{y}$ :

$$
\begin{aligned}
T_{x}= & 1+\pi \alpha v^{2} \sum_{k} \frac{1}{\alpha_{k}} \\
& \times\left\{f_{k}\left(T_{y}^{2} \Lambda^{2}\right) \frac{\Delta}{\alpha_{k}} T_{y}+i f_{k}\left(T_{x}^{2} \Lambda^{2}\right) X_{k}^{2} T_{x}\right\}, \\
T_{y}= & -\pi \alpha v^{2} \sum_{k} \frac{1}{\alpha_{k}} \\
& \times\left\{f_{k}\left(T_{x}^{2} \Lambda^{2}\right) \frac{\Delta}{\alpha_{k}} T_{x}-i f_{k}\left(T_{y}^{2} \Lambda^{2}\right) X_{k}^{2} T_{y}\right\},
\end{aligned}
$$

where $\alpha=e^{2} / \hbar c$ is the fine structure constant. The reflection coefficients are related to the transmission coefficients as $R_{x}=T_{x}-1$ and $R_{y}=T_{y}$. In the linear regime, these equations can be decoupled easily and reduce to the familar relations [9] for the transmission coefficients

$$
\begin{aligned}
& T_{x}=\frac{1+2 \pi \sigma_{x x} / c}{\left(1+2 \pi \sigma_{x x} / c\right)^{2}+\left(2 \pi \sigma_{y x} / c\right)^{2}}, \\
& T_{y}=-\frac{2 \pi \sigma_{y x} / c}{\left(1+2 \pi \sigma_{x x} / c\right)^{2}+\left(2 \pi \sigma_{y x} / c\right)^{2}},
\end{aligned}
$$

where $\sigma_{x x}$ and $\sigma_{y x}$ are respectively the longitudinal and Hall conductivities under RWA, given by Eqs. 201-21) through $\sigma_{x x}=\mathcal{J}_{x} / E_{0}$ and $\sigma_{y x}=\mathcal{J}_{y} / E_{0}$. In the strong field regime, Eqs. 27)-(28) must be solved simultaneously in order to obtain the transmission coefficients $T_{x, y}$. The Faraday $\theta_{F}$ and Kerr $\theta_{K}$ angles are connected in the usual way to the transmission and reflection coefficients as $\theta_{F}=\left[\arg \left(T_{-}\right)-\arg \left(T_{+}\right)\right] / 2$ and $\theta_{K}=\left[\arg \left(R_{-}\right)-\arg \left(R_{+}\right)\right] / 2$, where 'arg' denotes taking the complex argument, $T_{ \pm}=T_{x} \pm i T_{y}$ and $R_{ \pm}=R_{x} \pm i R_{y}$ stand for the transmission and reflection coefficients for the \pm circularly polarized components of the transmitted and reflected light, respectively.

Figs. 4.5 show the Faraday and Kerr angles calculated from numerically solving Eqs. 27)-28. The values approaching $E_{0}=0$ correspond to the results from weakfield regime where $T_{x, y}$ are independent of the incident field. One might naively expect that both angles increase with the electric field. On the contrary, we find that 


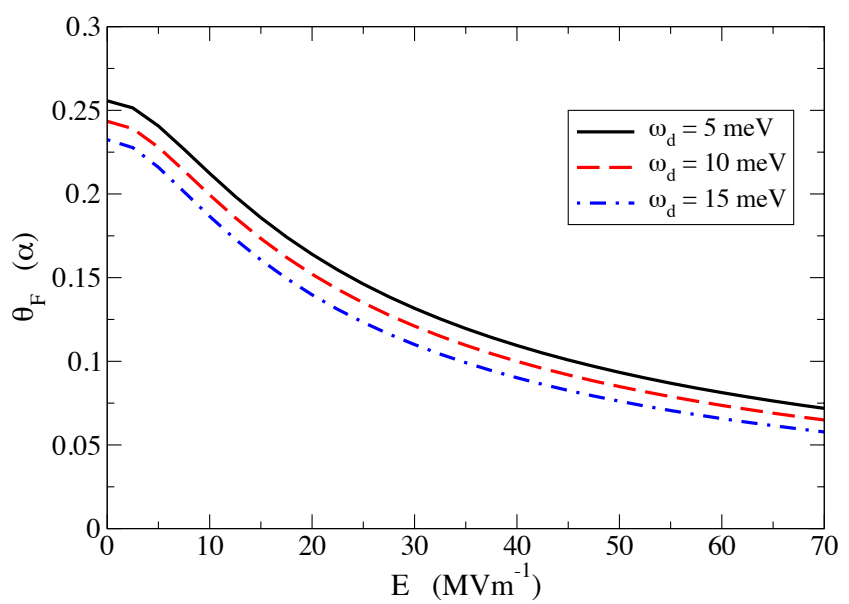

FIG. 4: (Color online) Faraday angle $\theta_{F}$ (in units of $\alpha$ radians, where $\alpha=1 / 137$ is the fine structure constant) versus electric field $E_{0}$ for different values of detuning $\omega_{\mathrm{d}}=5 \mathrm{meV}, 10 \mathrm{meV}$, $15 \mathrm{meV}$. The parameters $\Delta$ and $\varepsilon_{\mathrm{c}}$ are the same as that in Fig. 3 .

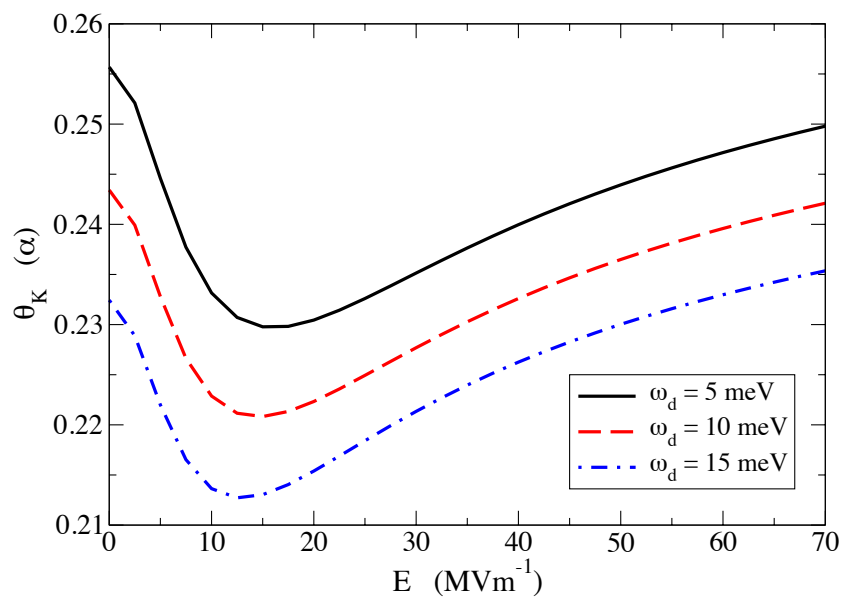

FIG. 5: (Color online) Kerr angle $\theta_{K}$ (in units of $\alpha$ radians) versus electric field $E_{0}$ for different values of detuning $\omega_{\mathrm{d}}=$ $5 \mathrm{meV}, 10 \mathrm{meV}, 15 \mathrm{meV}$.

$\theta_{F}$ decreases with the incident electric field whereas $\theta_{K}$ exhibits an interesting non-monotonic trend, decreasing first before going through an upturn. The leading-order nonlinear corrections for both effects are second order, a signature of optical Stark effect, going as $\theta_{F, K} \sim A-B E_{0}^{2}$ where $A, B$ are positive constants. We also note that both $\theta_{F}$ and $\theta_{K}$ increases with decreasing detuning.

\section{EFFECTS OF RELAXATION}

We now consider the regime when the frequency is close to the surface band gap with the detuning smaller than or on the same order of magnitude as the inverse lifetime $1 / \tau$ of the conduction electrons. To more fully characterize lifetime effects, we introduce the phenomenological longitudinal $\Gamma$ and transverse $\Gamma_{\perp}$ relaxation rates into the Bloch equation employing a relaxation time approximation. $\Gamma$ relaxes the conduction band electrons to the valence band due to momentum-independent processes such as radiative recombination and optical phonon scattering, whereas $\Gamma_{\perp}$ accounts for the decoherence effects on the polarization. Eq. (11) then becomes

$$
\begin{aligned}
& i \frac{\partial S_{k}^{\mathrm{L}}}{\partial t}=\frac{1}{2}\left[\left(S_{k}^{\mathrm{T}}\right)^{*} \mathcal{B}_{k}^{\mathrm{T}}-S_{k}^{\mathrm{T}}\left(\mathcal{B}_{k}^{\mathrm{T}}\right)^{*}\right]-i \Gamma\left(S_{k}^{\mathrm{L}}+1\right) \\
& i \frac{\partial S_{k}^{\mathrm{T}}}{\partial t}=\frac{1}{2}\left[S_{k}^{\mathrm{L}}\left(\mathcal{B}_{k}^{\mathrm{T}}\right)^{*}-\left(S_{k}^{\mathrm{T}}\right)^{*} \mathcal{B}_{k}^{\mathrm{L}}\right]-i \Gamma_{\perp} S_{k}^{\mathrm{T}}
\end{aligned}
$$

With damping, the spin density matrix no longer satisfy the unitarity condition $\left|\boldsymbol{S}_{k}\right|=1$ since the number of electrons in the system is not conserved. After the light field has been turned on and the transients have subsided, $\boldsymbol{S}_{k}$ approaches a steady-state value dependent on its initial state and the damping parameters $\Gamma, \Gamma_{\perp}$. The steady-state solution is obtained in the rotating frame by requiring, within RWA, that $\partial S_{k}^{\mathrm{L}, \mathrm{T}} / \partial t=0$. We then tranform the resulting expressions back into the stationary frame in order to obtain the currents from Eq. 15, yielding

$$
\begin{aligned}
\boldsymbol{S}_{k}= & -g_{k}(\Lambda)\left\{\left[\left(2 \alpha_{k}-\omega\right)^{2}+\Gamma_{\perp}^{2}\right] \hat{\boldsymbol{\alpha}}_{k}\right. \\
& +\left[\left(2 \alpha_{k}-\omega\right) \mathcal{R}_{k}^{\beta}+\Gamma_{\perp} \mathcal{R}_{k}^{\gamma}\right] \hat{\boldsymbol{\beta}}_{k} \\
& \left.+\left[\left(2 \alpha_{k}-\omega\right) \mathcal{R}_{k}^{\gamma}-\Gamma_{\perp} \mathcal{R}_{k}^{\beta}\right] \hat{\boldsymbol{\gamma}}_{k}\right\}
\end{aligned}
$$

where

$$
g_{k}(\Lambda)=\frac{1}{\left(2 \alpha_{k}-\omega\right)^{2}+\Gamma_{\perp}^{2}+\left(\Gamma_{\perp} / \Gamma\right)\left(\Lambda X_{k} / 2\right)^{2}} .
$$

Eqs. (33)- (34) are valid in the steady-state regime for nonzero $\Gamma, \Gamma_{\perp}$. Using Eq. (15), we then find the longitudinal and Hall current densities

$$
\begin{aligned}
& \mathcal{J}_{x}=i \frac{e^{2} E_{0} v^{2}}{2 \omega} \sum_{k} \frac{g_{k}(\Lambda)}{\alpha_{k}} X_{k}^{2}\left(\omega^{2}+\Gamma_{\perp}^{2}-2 \alpha_{k} \omega-i 2 \Gamma_{\perp} \alpha_{k}\right), \\
& \mathcal{J}_{y}=-\frac{e^{2} E_{0} v^{2} \Delta}{2 \omega} \sum_{k} \frac{g_{k}(\Lambda)}{\alpha_{k}^{2}}\left(\omega^{2}+\Gamma_{\perp}^{2}-2 \alpha_{k} \omega-i 2 \Gamma_{\perp} \alpha_{k}\right) .
\end{aligned}
$$

The presence of relaxation and dephasing introduces dissipation in the current response. In Eqs. (35)(36), $\operatorname{Im}\left\{\mathcal{J}_{x}\right\}$ and $\operatorname{Re}\left\{\mathcal{J}_{y}\right\}$ correspond to reactive, nondissipative current components whereas $\operatorname{Re}\left\{\mathcal{J}_{x}\right\}$ and $\operatorname{Im}\left\{\mathcal{J}_{y}\right\}$ correspond to dissipative components. 


\section{A. Weak Fields}

For $\Lambda \ll \delta \lesssim \Gamma, \Gamma_{\perp}$, we expand $\mathcal{J}_{x, y}$ in Eqs. $35-(36)$ to leading order in $\Lambda / \delta$ and obtain

$$
\begin{aligned}
\mathcal{J}_{x}= & -\frac{e^{2} E_{0}}{16 \pi \omega}\left\{\left(\omega+i \Gamma_{\perp}+\frac{4 \Delta^{2}}{\omega+i \Gamma_{\perp}}\right)\right. \\
& \times\left[\tan ^{-1}\left(\frac{\omega-2 \varepsilon_{c}}{\Gamma_{\perp}}\right)-\tan ^{-1}\left(\frac{\omega-2 \Delta}{\Gamma_{\perp}}\right)\right. \\
& \left.+\frac{i}{2} \ln \left[\frac{\left(\omega-2 \varepsilon_{c}\right)^{2}+\Gamma_{\perp}^{2}}{(\omega-2 \Delta)^{2}+\Gamma_{\perp}^{2}}\right]\right] \\
& \left.+i 2 \Delta^{2}\left[\frac{1}{\varepsilon_{c}}-\frac{1}{\Delta}-\frac{2}{\omega+i \Gamma_{\perp}} \ln \left(\frac{\varepsilon_{c}}{\Delta}\right)\right]\right\}, \quad 37 \\
\mathcal{J}_{y}=- & \frac{e^{2} E_{0} \Delta}{4 \pi \omega}\left\{\ln \left(\frac{\varepsilon_{c}}{\Delta}\right)-\frac{1}{2} \ln \left[\frac{\left(\omega-2 \varepsilon_{c}\right)^{2}+\Gamma_{\perp}^{2}}{(\omega-2 \Delta)^{2}+\Gamma_{\perp}^{2}}\right]\right. \\
+ & \left.i\left[\tan ^{-1}\left(\frac{\omega-2 \varepsilon_{c}}{\Gamma_{\perp}}\right)-\tan ^{-1}\left(\frac{\omega-2 \Delta}{\Gamma_{\perp}}\right)\right]\right\} .
\end{aligned}
$$

We note that the longitudinal relaxation rate $\Gamma$ does not come into the expressions Eqs. (37)-38 for $\mathcal{J}_{x, y}$. In the weak-field regime, therefore, relaxation processes such as radiative recombination and electron-optical phonon scattering that relax conduction band electrons back into the valence band do not contribute. This is because these processes only happen when there are real transitions resulting in a finite electron population in the conduction band. For subgap frequencies, a finite electron population in the conduction band only occurs through nonlinear correction $\sim E_{0}^{2}$, and are absent in the linear $\sim E_{0}$ regime.

Despite $\Gamma, \Gamma_{\perp}>0$ in the regime considered in this section, we note that the $\Gamma_{\perp} \rightarrow 0$ limit of Eqs. (37)-(38) recovers Eqs. 200-21) obtained in the coherent regime where the effects of damping are ignored. This correspondence however is restricted only to the linear regime, and does not hold when nonlinear effects in the electric field come in.

\section{B. Strong Fields}

For strong fields $\Lambda \gg \delta, \Gamma, \Gamma_{\perp}$, we have

$$
g_{k}(\Lambda) \simeq \frac{4 \Gamma}{\Gamma_{\perp} \Lambda^{2}} \frac{1}{X_{k}^{2}}
$$

Eqs. (35)-36 can then be evaluated analytically yielding

$$
\begin{aligned}
\mathcal{J}_{x}= & \frac{i \omega}{4 \pi E_{0} v^{2}} \frac{\Gamma}{\Gamma_{\perp}}\left(\varepsilon_{c}-\Delta\right)\left(\omega+i \Gamma_{\perp}\right) \\
& \times\left(\omega-i \Gamma_{\perp}-\varepsilon_{c}-\Delta\right) \\
\mathcal{J}_{y}= & -\frac{\omega}{4 \pi E_{0} v^{2}} \frac{\Gamma}{\Gamma_{\perp}}\left(\varepsilon_{c}-\Delta\right)\left(\omega+i \Gamma_{\perp}\right) \\
& \times\left(\omega-i \Gamma_{\perp}-\varepsilon_{c}-\Delta\right)
\end{aligned}
$$

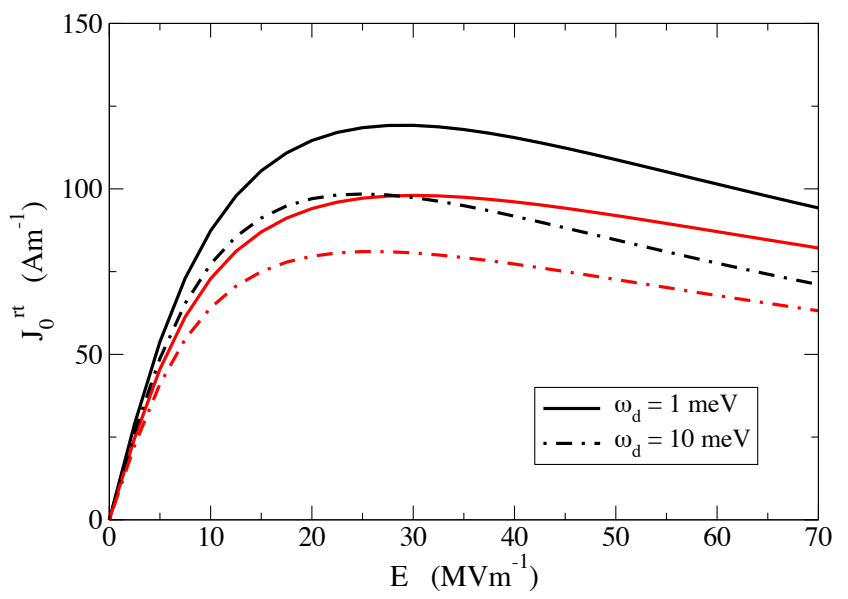

FIG. 6: (Color online) Amplitudes of the reactive components (denoted by the superscript 'rt') $J_{x 0}^{\text {rt }}=\operatorname{Im}\left\{\mathcal{J}_{x}\right\}$ (dark/black) and $J_{y 0}^{\text {rt }}=\operatorname{Re}\left\{\mathcal{J}_{y}\right\}$ (grey/red) of the longitudinal and Hall current densities, as a function of the electric field $E_{0}$ for $\Gamma=\Gamma_{\perp}=5 \mathrm{meV}$ and different values of detuning $\omega_{\mathrm{d}}=1 \mathrm{meV}$ and $10 \mathrm{meV}$. The values of the surface Dirac gap and Dirac band cutoff energy are the same as in Fig. 3

In contrast to the results we find in the coherent regime where both the longitudinal and Hall currents saturate at high $E_{0}$ values, in our present case we find that $\mathcal{J}_{x, y}$ do not saturate but instead decrease at high electric fields as $1 / E_{0}$. Fig. 6 shows the amplitudes of the reactive components of the longitudinal and Hall current densities calculated from Eqs. (35)-36. Comparing the results for $\omega_{\mathrm{d}}=10 \mathrm{meV}$ in the absence and presence of $\Gamma, \Gamma_{\perp}$ (Fig. 3 and Fig. 6, respectively), we see that relaxation and dephasing decrease the reactive components of the longitudinal and Hall currents. In the regime considered where $\delta \lesssim \Gamma, \Gamma_{\perp}$, the dissipative current components are finite but still small, about an order of magnitude less than that of the reactive components (Fig. 7).

\section{Nonlinear Faraday and Kerr Effects}

Including relaxation and dephasing rates, we find that the coupled equations for $T_{x}$ and $T_{y}$ are modified as follows

$$
\begin{aligned}
T_{x}= & 1-\frac{\pi \alpha v^{2}}{\omega} \sum_{k} \frac{\omega^{2}+\Gamma_{\perp}^{2}-2 \alpha_{k} \omega-i 2 \Gamma_{\perp} \alpha_{k}}{\alpha_{k}} \\
& \times\left\{g_{k}\left(T_{y}^{2} \Lambda^{2}\right) \frac{\Delta}{\alpha_{k}} T_{y}+i g_{k}\left(T_{x}^{2} \Lambda^{2}\right) X_{k}^{2} T_{x}\right\} \\
T_{y}= & \frac{\pi \alpha v^{2}}{\omega} \sum_{k} \frac{\omega^{2}+\Gamma_{\perp}^{2}-2 \alpha_{k} \omega-i 2 \Gamma_{\perp} \alpha_{k}}{\alpha_{k}} \\
& \times\left\{g_{k}\left(T_{x}^{2} \Lambda^{2}\right) \frac{\Delta}{\alpha_{k}} T_{x}-i g_{k}\left(T_{y}^{2} \Lambda^{2}\right) X_{k}^{2} T_{y}\right\} .
\end{aligned}
$$

Figs. 8.9 show the calculated Faraday and Kerr rotations from the numerical solutions of Eqs. 42. 43 . 


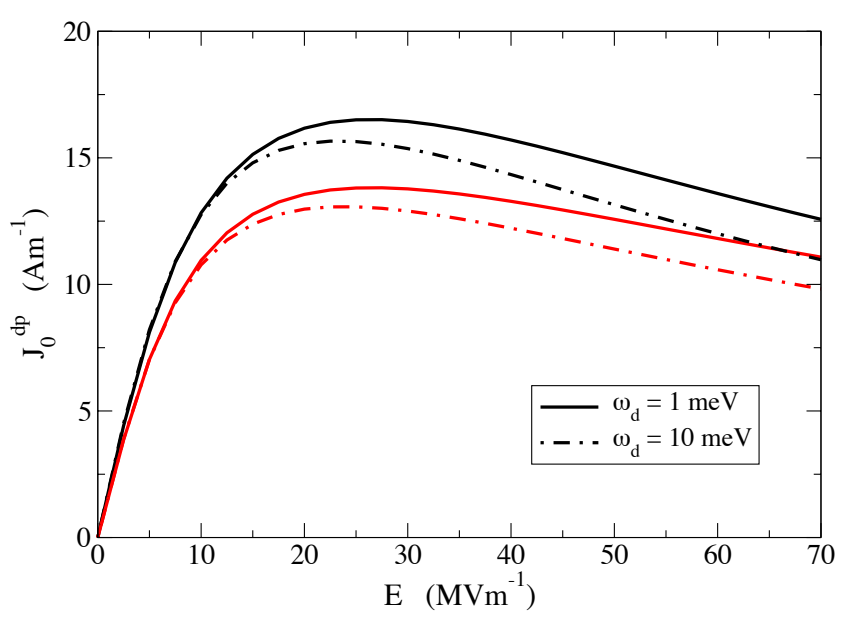

FIG. 7: (Color online) Amplitudes of the dissipative components (denoted by the superscript 'dp') $J_{x 0}^{\text {dp }}=\operatorname{Re}\left\{\mathcal{J}_{x}^{\text {dp }}\right\}$ (dark/black) and $J_{y 0}^{\text {dp }}=\operatorname{Im}\left\{\mathcal{J}_{y}^{\text {dp }}\right\}$ (grey/red) of the longitudinal and Hall current densities, as a function of the electric field $E_{0}$ for $\Gamma=\Gamma_{\perp}=5 \mathrm{meV}$ and different values of detuning $\omega_{\mathrm{d}}=1 \mathrm{meV}$ and $10 \mathrm{meV}$.

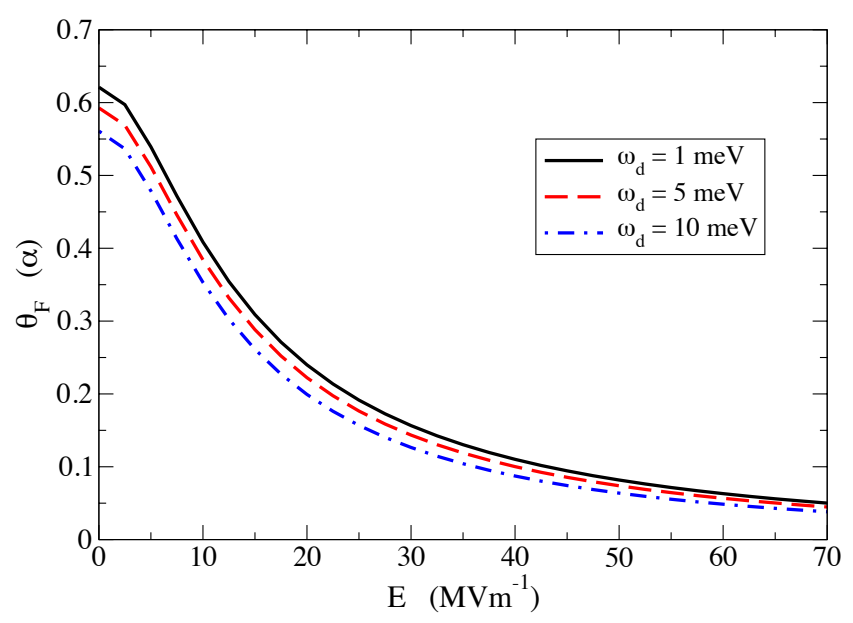

FIG. 8: (Color online) Faraday angle $\theta_{F}$ (in units of $\alpha$ radians) versus electric field $E_{0}$ for $\Gamma=\Gamma_{\perp}=5 \mathrm{meV}$ and different values of detuning $\omega_{\mathrm{d}}=1 \mathrm{meV}, 5 \mathrm{meV}, 10 \mathrm{meV}$. The parameters $\Delta$ and $\varepsilon_{\mathrm{c}}$ are the same as that in Fig. 3

The decreasing trend of the Faraday angle and the nonmonotonic behavior of the Kerr angle with increasing electric field remain similar to the coherent case. Although the reactive components of $\mathcal{J}_{x}$ and $\mathcal{J}_{y}$ are reduced by relaxation (c.f. Fig. 3 and Fig. 6), we find that, interestingly, the Faraday and Kerr effects are enhanced in the presence of relaxation and dephasing compared to the coherent case (Figs. 445). In particular, $\theta_{K}$ is enhanced by an order of magnitude and is more sensitive than $\theta_{F}$ to increasing values of $\Gamma$ and $\Gamma_{\perp}$, as depicted in Fig. 10. To a lesser extent, this behavior is also observed when $\theta_{K}$ is varied as a function of detuning $\omega_{d}$.

To exhibit this curious enhancement and sensitivity

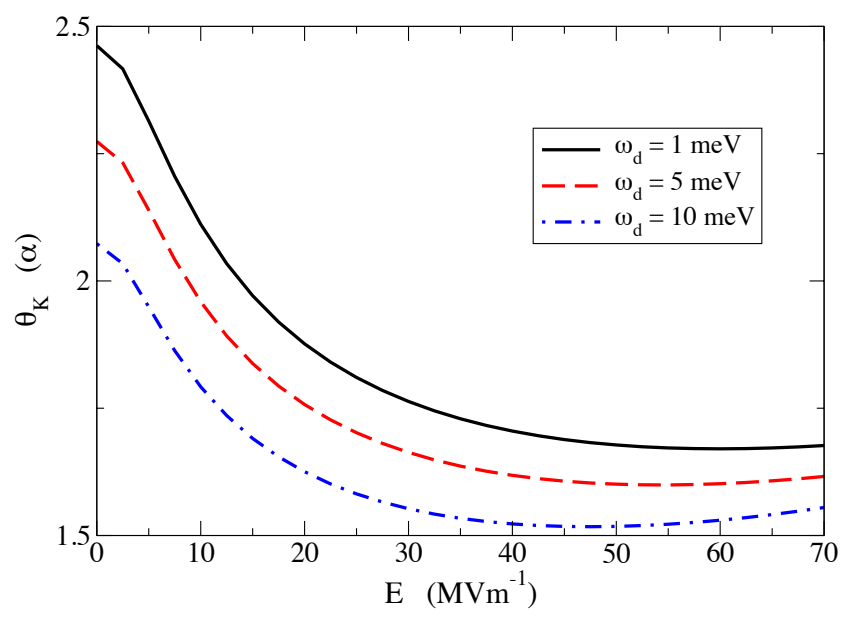

FIG. 9: (Color online) Kerr angle $\theta_{K}$ (in units of $\alpha$ radians) versus electric field $E_{0}$ for $\Gamma=\Gamma_{\perp}=5 \mathrm{meV}$ and different values of detuning $\omega_{\mathrm{d}}=1 \mathrm{meV}, 5 \mathrm{meV}, 10 \mathrm{meV}$.

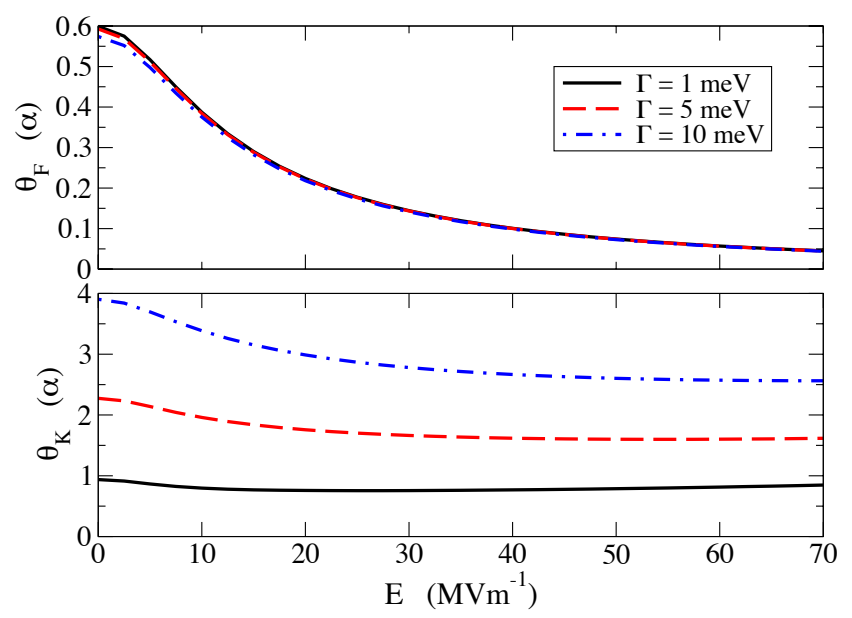

FIG. 10: (Color online) Faraday $\theta_{F}$ and Kerr $\theta_{K}$ angles (in units of $\alpha$ radians) versus electric field $E_{0}$ for detuning $\omega_{\mathrm{d}}=$ $5 \mathrm{meV}$ and different values of $\Gamma=\Gamma_{\perp}=1,5,10 \mathrm{meV}$.

of $\theta_{K}$ over $\theta_{F}$, we consider the weak-field regime when nonlinear effects are absent and evaluate the Faraday and Kerr angles up to leading order in $\alpha=e^{2} / \hbar c$. From Eqs. 29)-(30) we have $T_{x} \simeq 1-2 \pi \sigma_{x x} / c, T_{y} \simeq$ $-2 \pi \sigma_{y x} / c$, and $R_{x} \simeq-2 \pi \sigma_{x x} / c, R_{y} \simeq-2 \pi \sigma_{y x} / c$, where $\sigma_{x x}, \sigma_{y x}$ are the longitudinal and Hall conductivities corresponding to Eqs. (37)-(38). For the transmitted light, $\operatorname{Re}\left\{T_{ \pm}\right\} \approx 1+\mathcal{O}\left(\alpha^{1}\right)$ and $\operatorname{Im}\left\{T_{ \pm}\right\} \approx \mathcal{O}\left(\alpha^{1}\right)$ imply that $\theta_{F} \approx \tan ^{-1}\left[\mathcal{O}\left(\alpha^{1}\right)\right]$. For the reflected light, however, we have both $\operatorname{Re}\left\{R_{ \pm}\right\}, \operatorname{Im}\left\{R_{ \pm}\right\} \approx \mathcal{O}\left(\alpha^{1}\right)$ yielding $\theta_{K} \approx \tan ^{-1}\left[\mathcal{O}\left(\alpha^{0}\right)\right]$. The Kerr angle is therefore larger than the Faraday angle by about an order of magnitude, resulting in the larger enhancement and increased sensitivity with changes in relaxation rate and detuning. 


\section{CONCLUSION}

In summary, we have developed a theory for the magneto-optical effects in topological insulators under intense laser fields in the small detuning regime $\delta \ll \Delta$. We calculated the nonlinear longitudinal and Hall currents in response to linearly polarized light and obtained the Faraday and Kerr rotations as a function of the incident electric field. Surprisingly, damping effects due to relaxation and dephasing are found to enhance the resulting Faraday and Kerr rotations. In particular, the Kerr effect exhibits a larger enhancement and higher sensitivity to changes in detuning and damping rate than the Faraday effect. As limiting cases, we examined the current responses in the weak field and strong field regimes. In the weak field regime, the currents we obtained under rotating-wave approximation account exactly for the resonant contribution in linear response theory. In the strong field regime, no such correspondence can be found. The currents saturate for negligible damping but decrease with electric field when damping is taken into account. The leading-order nonlinear dependence of the Faraday and Kerr rotations on the incident field implies that optical Stark effect can be probed using magneto-optical spectroscopy. Although we focused on the case of a topological insulator surface, our results also carry over directly to $2 \mathrm{D}$ Chern insulators, whose low-energy theory is described similarly by the 2D Dirac model.

\section{ACKNOWLEDGEMENT}

The author acknowledges useful discussions with R. Binder. This work is supported by a startup fund from the University of Alabama.

\section{APPENDIX}

\section{A. Spin Representation}

The expressions for the spin vectors defined in Sec. II are

$$
\begin{aligned}
\hat{\boldsymbol{\alpha}}_{k} & =\sin \theta_{k} \hat{\boldsymbol{k}}+\cos \theta_{k} \hat{\boldsymbol{z}}, \\
\hat{\boldsymbol{\beta}}_{k} & =\hat{\boldsymbol{z}} \times \hat{\boldsymbol{k}} \\
\hat{\boldsymbol{\gamma}}_{k} & =-\cos \theta_{k} \hat{\boldsymbol{k}}+\sin \theta_{k} \hat{\boldsymbol{z}}
\end{aligned}
$$

This gives the following longitudinal and transverse spin vectors defined in Sec. III

$$
\begin{aligned}
\hat{\boldsymbol{\alpha}}_{k}^{\mathrm{L}} & =\sin \theta_{k} \hat{\boldsymbol{k}}+\cos \theta_{k} \hat{\boldsymbol{z}}, \\
\hat{\boldsymbol{\alpha}}_{k}^{\mathrm{T}} & =\hat{\boldsymbol{z}} \times \hat{\boldsymbol{k}}+i \cos \theta_{k} \hat{\boldsymbol{k}}-i \sin \theta_{k} \hat{\boldsymbol{z}},
\end{aligned}
$$

where $\hat{\boldsymbol{k}}=\cos \phi_{k} \hat{\boldsymbol{x}}+\sin \phi_{k} \hat{\boldsymbol{y}}$ is the unit momentum vector with an azimuthal angle $\phi_{k}$.
[1] L. Fu, C. L. Kane, and E. J. Mele, Phys. Rev. Lett. 98, 106803 (2007); J. E. Moore and L. Balents, Phys. Rev. B 75, 121306(R) (2007); R. Roy, Phys. Rev. B 79, 195321 (2009).

[2] M. Z. Hasan and C. L. Kane, Rev. Mod. Phys. 82, 3045 (2010); X.-L. Qi and S.-C. Zhang, Rev. Mod. Phys. 83, 1057 (2011).

[3] X.-L. Qi, T. L. Hughes, and S.-C. Zhang, Phys. Rev. B 78, 195424 (2008).

[4] A. M. Essin, J. E. Moore, and D. Vanderbilt, Phys. Rev. Lett. 102, 146805 (2009).

[5] J. Maciejko, X.-L. Qi, H. D. Drew, and S.-C. Zhang, Phys. Rev. Lett. 105, 166803 (2010).

[6] F. Wilczek, Phys. Rev. Lett. 58, 1799 (1987).

[7] W.-K. Tse and A. H. MacDonald, Phys. Rev. Lett. 105, 057401 (2010).

[8] W.-K. Tse and A. H. MacDonald, Phys. Rev. B 82, 161104(R) (2010).

[9] W.-K. Tse and A. H. MacDonald, Phys. Rev. B 84, 205327 (2011).

[10] R. Valdés Aguilar, A. V. Stier, W. Liu, L. S. Bilbro, D. K. George, N. Bansal, J. Cerne, A. G. Markelz, S. Oh, and N. P. Armitage, Phys. Rev. Lett. 108, 087403 (2012).
[11] L. Wu, W.-K. Tse, M. Brahlek, C. M. Morris, R. Valds Aguilar, N. Koirala, S. Oh, N. P. Armitage, Phys. Rev. Lett. 115, 217602 (2015).

[12] K. N. Okada, Y. Takahashi, M. Mogi, R. Yoshimi, A. Tsukazaki, K. S. Takahashi, N. Ogawa, M. Kawasaki, Y. Tokura, arXiv:1603.02113v1 (2016).

[13] L. Wu, M. Salehi, N. Koirala, J. Moon, S. Oh, N. P. Armitage, arXiv:1603.04317v1 (2016).

[14] V. Dziom, A. Shuvaev, A. Pimenov, G. V. Astakhov, C. Ames, K. Bendias, J. Bttcher, G. Tkachov, E. M. Hankiewicz, C. Brne, H Buhmann, L. W. Molenkamp, arXiv:1603.05482 1 (2016).

[15] See, for example, H. Haug and A. P. Jauho, Quantum Kinetics in Transport and Optics of Semiconductors $\left(2^{\text {nd }}\right.$ Ed., Springer-Verlag Berlin Heidelberg, 2008) and references therein.

[16] V. M. Galitskii, S. P. Goreslavskii, and V. F. Elesin, Sov. Phys.-JETP 30, 117 (1970).

[17] S. Schmitt-Rink, D. S. Chemla, and H. Haug, Phys. Rev. B 37, 941 (1988); R. Cote and H. Haug, Phys. Rev. B 40, 3802 (1989); S. Glutsch and R. Zimmermann, Phys. Rev. B 45, 5857 (1992). 